\title{
A case of drug-coated balloon treatment for two total occluded lesions in a patient with acute coronary syndrome
}

\author{
Song Lin Yuan ${ }^{1,2}$, Moo Hyun Kim² ${ }^{2}$ Scot Garg ${ }^{3}$, Eun-Seok Shin ${ }^{1}$ \\ ${ }^{1}$ Division of Cardiology, Department of Internal Medicine, Ulsan Medical Center, Ulsan, South Korea \\ ${ }^{2}$ Department of Cardiology, Dong-A University Hospital, Busan, South Korea \\ ${ }^{3}$ East Lancashire Hospitals NHS Trust, Blackburn, Lancashire, United Kingdom
}

A 51-year-old man was admitted with worsening effort angina over a 1 month period. His only coronary risk factor was hypercholesterolemia. Cardiac enzymes including creatine kinase-MB and troponin I were normal. His electrocardiogram showed Q-waves in $\mathrm{V}_{1}$ to $\mathrm{V}_{4}$. The coronary angiograms showed a short left main with complete occlusions in the proximal left anterior descending (LAD) and left circumflex (LCX) artery (Fig. 1A-C). Revascularization options were discussed and the option to treat the lesions with drug-coated balloons (DCB) was decided. He was carefully assessed, and gave informed consent.

A guide wire successfully crossed the total occlusion of the LCX. Pre-dilatation was performed with a $1.5 \times 15 \mathrm{~mm}$ balloon, followed by a $2.5 \times$ $\times 15 \mathrm{~mm}$ non-compliant balloon up to $16 \mathrm{~atm}$ and finally a $2.5 \times 30 \mathrm{~mm}$ DCB was inflated at $7 \mathrm{~atm}$ for $60 \mathrm{~s}$. Then to the LAD lesion, pre-dilatation was performed with $1.5 \times 15 \mathrm{~mm}$ balloon and a $3.0 \times$ $\times 15 \mathrm{~mm}$ scoring balloon at $16 \mathrm{~atm}$, and then finally a $3.5 \times 20 \mathrm{~mm}$ DCB was inflated at 7 atm for $60 \mathrm{~s}$. The final angiograms showed normal flow in both LAD and LCX with no significant dissection or residual stenoses (Fig. 1D-F).

Three months later, follow-up coronary angiography confirmed adequate patency of the DCB treated lesions and reassuringly the distal LCX and proximal LAD looked better (Fig. 1G-I). He remains symptom free, 13-month post-intervention.

After treatment with a DCB, it is possible that vessels will return to their original size over time, which is one of the greatest advantages of $\mathrm{DCB}$ treatment in total occluded lesions.

Conflict of interest: None declared

Address for correspondence: Eun-Seok Shin, MD, PhD, Division of Cardiology, Department of Internal Medicine, Ulsan Medical Center, Ulsan, South Korea, e-mail: sesim1989@gmail.com 


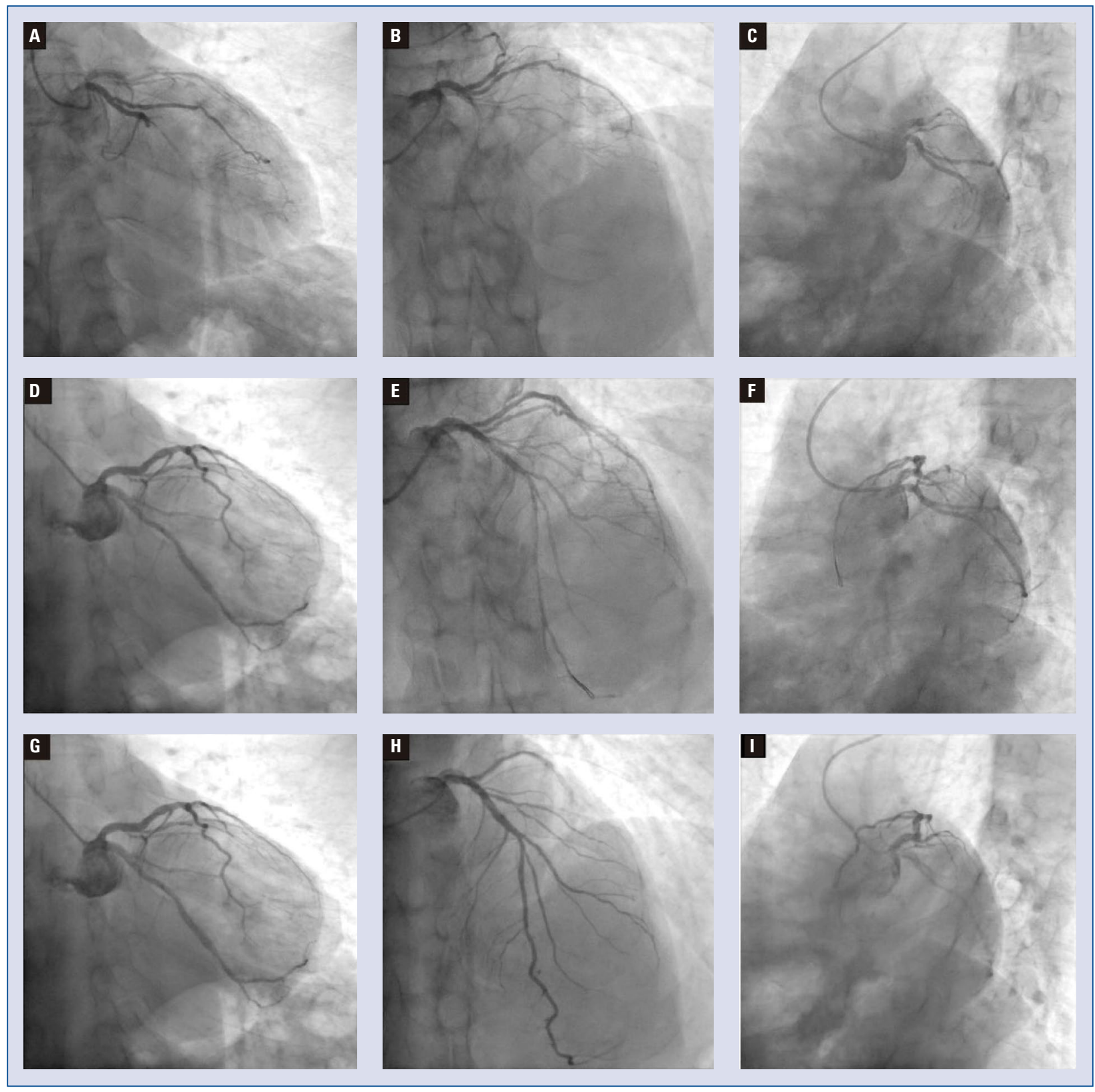

Figure 1. Coronary artery angiography; A-C. Before intervention; D-F. Right after treatment with a drug-coated balloon; G-I. Follow-up angiography at 3 months. 